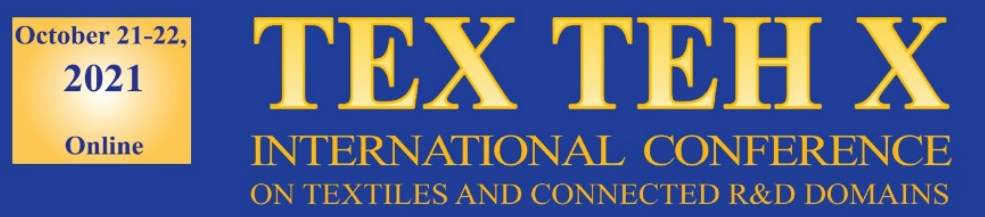

\title{
VIRTUAL 3D KINEMATIC HUMAN MODEL PROTOTYPE
}

DOI: 10.35530/TT.2021.35

\author{
D.R. Viziteu, A. Curteza, M.L. Avadanei \\ “Gheorghe Asachi” Technical University of Iasi, Doctoral School - Faculty of Industrial Design and \\ Business Management, Iasi, Romania \\ (E-mail: viziteudianaroxana@gmail.com, acurteza@gmail.com,mavad@tex.tuiasi.ro)
}

\begin{abstract}
In the past several years, the application of $3 D$ technologies in the textile and clothing design industry has considerably increased and become more accessible to designers and patternmakers. With digitisation in garment engineering and virtual prototype and modelling techniques becoming more mainstream, a new generation of virtual human models starts to develop to fulfil the demand for protective and functional products designed for specific athletes, such as climbers and mountaineers. We must base our work on an improved understanding of the behaviour of the musculoskeletal system to develop garment patterns that minimise discomfort and improve performance under dynamic body deformations and muscle contractions associated with specific movements. For this study, we explored the possibilities of using existing software packages for virtual prototyping based on human kinematic models for functional clothing.
\end{abstract}

Keywords: clothing design, digitalization, kinematic human model, virtual prototyping

\section{INTRODUCTION}

Patternmakers are still developing clothes based on the standard anthropometric static protocol defined by ergonomic standards or size charts: standing and looking straight ahead, with shoulders relaxed, arms at sides, and legs hip widespread [1]. When the size charts do not cover the requirements of personal protective equipment, there are measurements during movements as standing, sitting, kneeling, etc. [2]. Since the human body is engaged in permanent movement, and the demand for functional garments for work or sportswear is a modern concern, understanding how the human body alters in dynamic is an essential and contemporary research topic.

In current practice, dynamic anthropometry in the clothing industry was first applied for functional and aesthetic sportswear products [3]. Because functional clothing, especially for sports athletes, has complex requirements regarding increased physical comfort, performance, ease, and range of motion, the $2 \mathrm{D}$ flat pattern methods fail to capture the complex form of the human body shape in movement. In this case, an efficient method that can enhance body mobility and fitting test in movement is using 3D human body data and 3D technology in order to develop clothing.

Several changes have been implemented to bring virtual humans closer to our everyday lives in the past years. These human body models can be found in many interactive applications for virtual reality (VR) and augmented reality (AR), including games, movies or commercials. Virtual prototyping is usually based on avatars, garment patterns and textile materials. In the garment industry, most virtual models use realistic virtual representations of humans based on 3D anatomy scan data or used to the body measurement standard charts. Commercially available software package for garment 
industry that offers avatars includes CLO 3D by CLO Virtual Fashion, Vidya by Assyst, V Stitcher by Browzwear Solutions, Modaris by Lectra and various others.

Morlock et al. were the first to investigate the differences between body measurements for work or sports associated positions for both men and women [4]. 3D garment simulation software can be used for fit assessment in dynamic positions for garments developed for work or sportswear.

We can mention various skin deformation software avatars such as:

- Linear Blend Skinning - (LBS) - standard and popular method where the deformation of the character skin is driven via an underlying skeleton. The linear models of male and female body shape are obtained from the CAESAR dataset [5] (approximately 2000 scans per gender) [6][7];

- Skinned Multi-Person Linear - (SMPL) - skinned vertex-based model (male, female, gender-neutral) that precisely characterises a wide variety of body shapes in real human poses, from thousands of 3D body scans [8];

- Dynamic Multi-Person Linear - (DMPL) - an extended version of SMPL model that captures soft-tissue in dynamic [9];

- Bone-Level Skinned Model (BLSM) - the bone scales are set prior to template synthesis; the human body mesh first sets bone lengths and joint angles to specify the skeleton [10].

Researchers proposed a method based on the BLSM model, capable of precisely reconstructing 3D human body pose in the outdoor environment [11]. Klepser et. al [12], compared avatars from three 3D garment simulation software systems (CLO 3D, VStitcher, Vidya) with 4D scans of real test persons, revealing their limitations, especially in movement. Some authors have synthesised that 3D product development based on the kinematic human body and 3D technology is an efficient way to create close-fitting clothes straight on the body. Kanika et al. [13] proposed an efficient method to design functional clothing, developing virtual 3D patterns for specific body postures of a motorcycle rider. We considered the human kinematic models presented in the literature, which can be combined with different software applications into the digital process to develop functional garments [14].

\section{MATERIALS AND METHODS}

The garment simulation method is usually performed in the following stages: an avatar is selected according to defined body measurements, the pattern parts are assembled at the seams and digitally sewn together, the garment is placed and draped on the avatar, the properties of the fabric are defined, fit assessment and evaluation of the model.

Body movement is recorded by motion-capture technology in other fields such as video game design, animation, virtual reality, recuperative pain medicine and sport. There are different methods for studying body motion having the same fundamental principle of triangulation - e.g., 3D scanners, photogrammetry software, inertial sensors, etc.

Kinematics, in this paper, is an interdisciplinary field that analyses human movement. It describes the motion of points, objects and systems of groups of objects without reference to the causes of motion (e.g., forces). The study of kinematics is often referred to as the "geometry of motion". Through the lens of kinematics, we must consider information about the movement of the body, limbs and joints, that occurs during rock climbing [15]. There are big variances in geometry between the genders in body forms, proportions and muscle manifestations. In most cases, the kinematic 3D model is built of skeleton, muscles and skin. 
The sport of rock climbing or bouldering involves both lower and upper limbs, with movement between rests (figure 1). There are different phases in climbing on a rock surface, through continuous upward body movements, such as posture equilibrium, arm release, climbing-specific foot raise, resting and another route-finding $[16,17]$. The climber's success is based on the skill of moving fluidly between the rest points while maintaining the centre of gravity over one or, depending on the given route, on both feet. Rock climbing is interesting and complex because of the unequal spreading of the holds that come in so many angles and sizes. Based on that, the athlete has to perform a complex form of locomotion with extreme and contorted postures, combining key physiological components like power, strength and muscular endurance at variable angles.

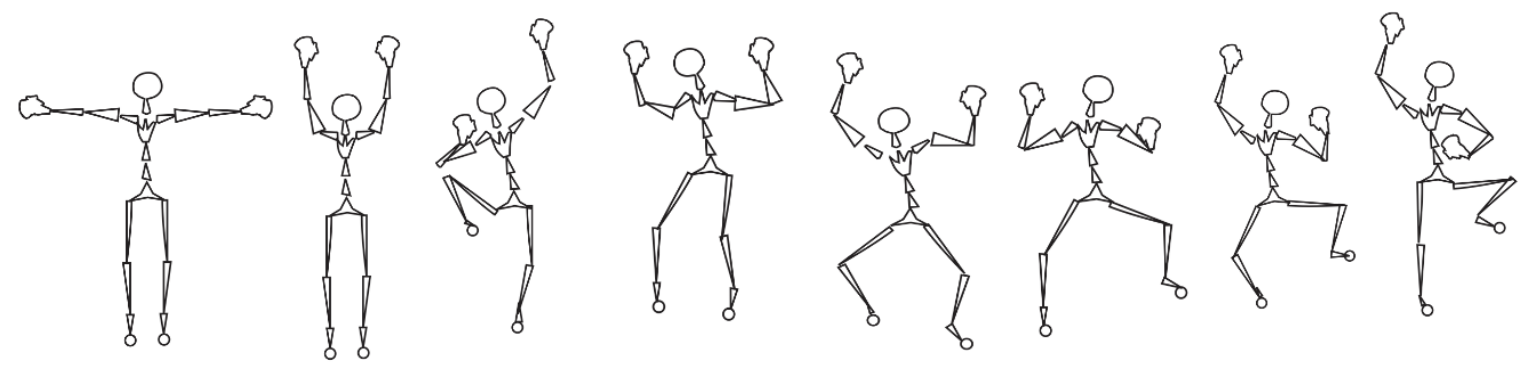

Figure 1. Climbing movements synthesised on body skeleton

The simulation was performed using a generated male muscular avatar revised to define body measurements [18]. In our case, we considered that muscle deformation is an important design component in clothes deformation. We integrated the avatar into auto-rigging online service Mixamo to insert animation structures from joints and bones, similar to those from rock climbing [19]. We placed the markers on the avatar joint positions for chin, wrists, elbows, knees and groin (figure 2).

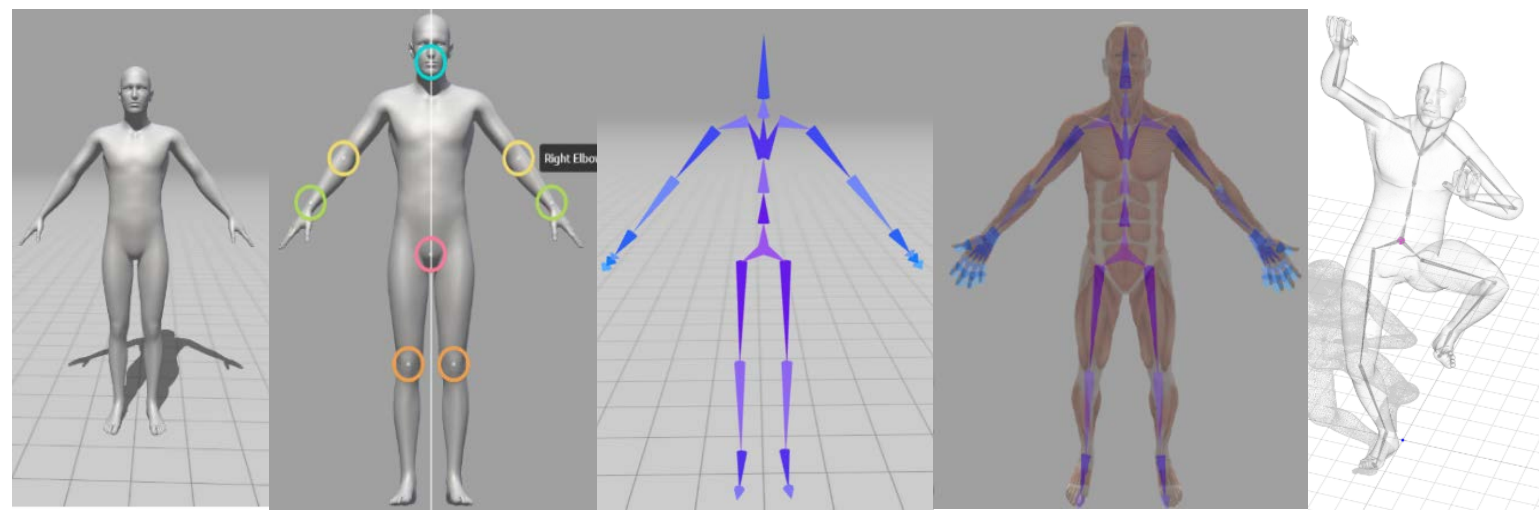

Figure 2. Auto-rigging: 3d body surface, place makers on joints, inserts skeleton generation of animated $3 \mathrm{~d}$ avatar

The skeleton can be used as a framework for scan data. To test the interaction between the animated avatar and the virtual garment prototype for fitting, we used Clo3d [20]. The auto-rigged avatar is imported in FBX format. The rigged avatars can be utilised across the current platforms for garment simulation.

\section{RESULTS AND DISCUSSIONS}

3D virtual prototyping of garment products enables the designers to easily see how their new or existing 2D pattern will look with a wide range of materials and diverse strain situations. Additionally, 3D animation with apparel design can be used in e- 
commerce. Digitalisation in garment development involves applying a great diversity of CAD systems with integrated 3D modules to develop the pattern designs and assess their fit to the 3D body model. The great advantage is that we can check the fit simulation and the virtual wearing comfort of functional clothing by using pressure tools and analysing stress, pressure points, strain and fit maps.

We tested the animated dummy as an import into Clo3d and designed clothing on it. Examining avatars exposes alterations between software in appearance and shape. Various methods to study 3D garment simulation were reviewed. The obtained results are:

(1) identifying climbing phases through kinematic analysis;

(2) generating a rigged avatar;

(3) obtaining postures with muscle deformation;

(4) case I - garment pattern construction using the traditional 2D method, then analyse fit in motion;

(5) case II - garment pattern created with 3D flattening technique direct on dynamic posture;

(6) findings.

The postures obtained from the generated rigged avatar are reliable in considering which areas of the body are exposed to extreme tension. The kinematic analysis showed that the knee, hip and crotch region are the stress points during bouldering specific movements.

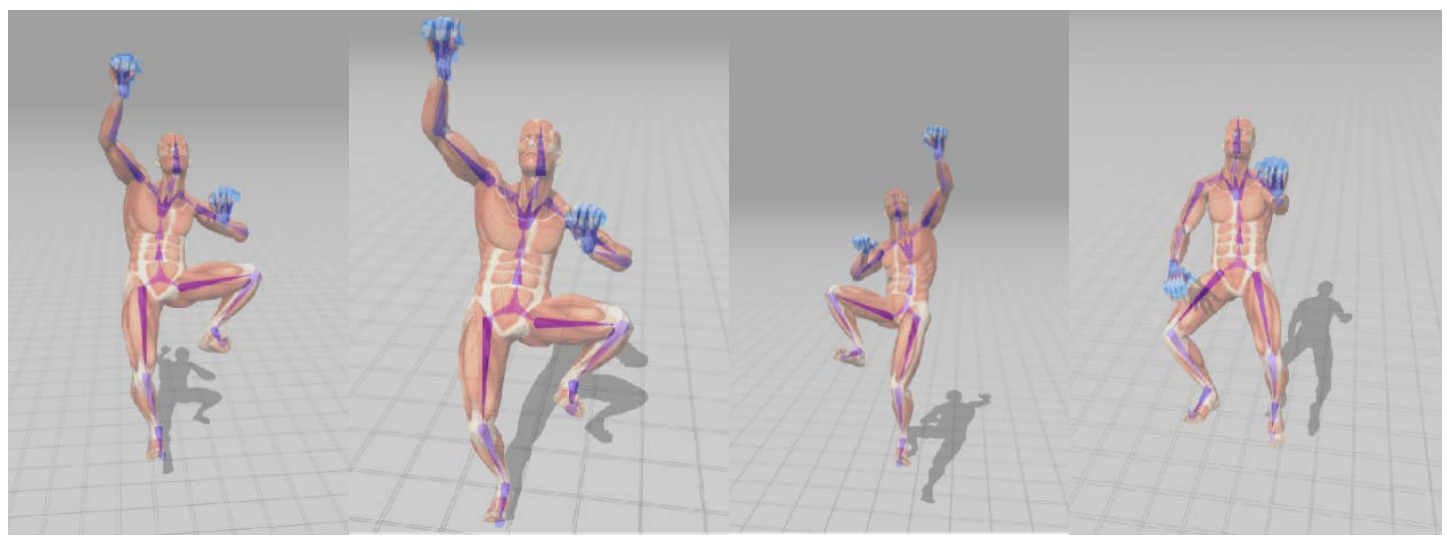

Figure 3. Postures with muscle deformation

In case I, starting from the avatar static posture, we adopted the traditional 2D method of garment pattern construction for outdoor climbing pants, animated in Mixamo and then analysed fit in motion. We observed that the dressed avatar in motion shows a disturbing effect at the hip and knee joints (figure 4).

The garment pattern for the second case of outdoor pants design will consider the mapped red zones on the avatar (figure 4). We can observe muscle deformation during the knee and hip flexion, where, normally, the fabric should extend the same as the muscle will contract during bouldering movement, being like a second skin. Following the avatar shape from the dynamic posture, we can trace the body skin deformation to create lines and shapes (figure 5). The lines are drawn with the 3D Pen (Avatar) directly on the virtual climber. It is essential to draw and close shapes on the avatar because the flattening function only recognises and extracts closed surfaces as patterns. 

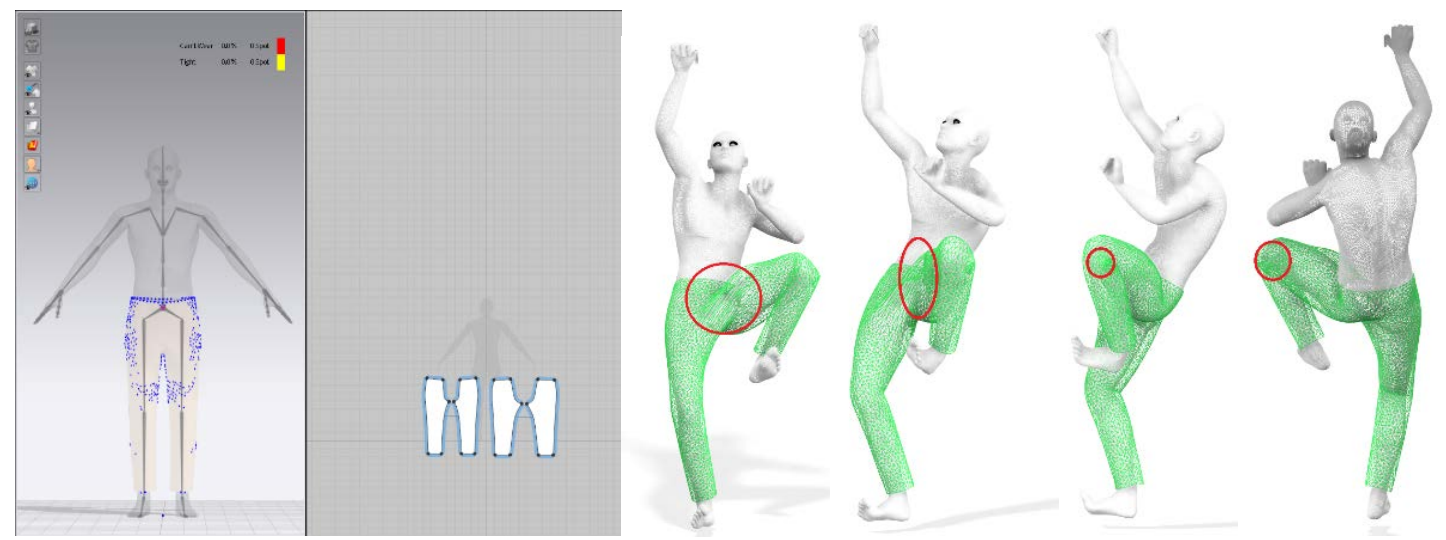

Figure 4. Case I - 3D simulation and fit analysis
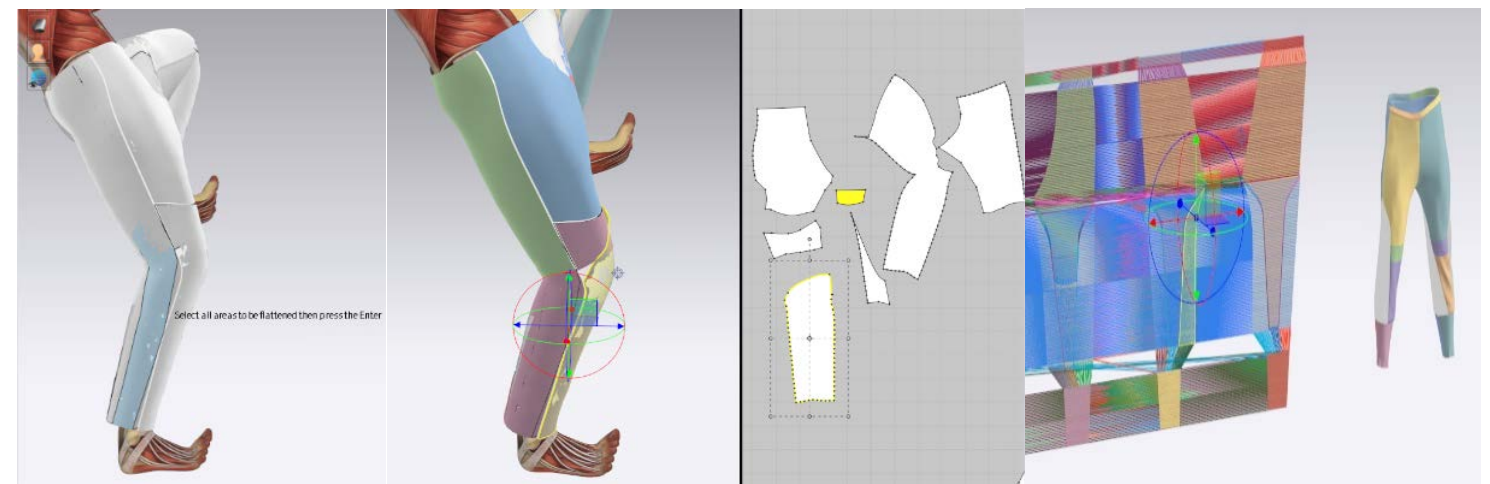

Figure 5. Case II - Pattern flattening and 3D prototype

When designing garments alongside 3D avatars, we can test the fit against flexed limbs and muscle bunches in real-time and dynamic motion, which vary the diameter and overall shape of limbs. In these situations, it is much clearer where garments need to be elastic or have fabric folds that compensate for dynamic shape differences between static and dynamic states of the body. While avatars are a very useful emergent tool in garment design, one must consider the shortcomings of incipient states of technology - e.g., the first avatars somewhat struggle to recreate soft tissue folds along large joins. We must further include and update 3D models with anthropometric parameter data sets to achieve a 100\% accurate, complete form-fitting dynamic mannequin. There has been interesting in the perfection of 3D fabric dynamic and draping on scans and models. The area lacking is making the actual dynamic models accurate, taking into account scan data and adapting to it. Hence, this paper aims to assess virtual prototyping using personalised kinematic human models.

\section{CONCLUSION}

Animating digital humans with clothes has numerous applications, especially with 3D content for fashion runaway collections, e-commerce, entertainment or virtual try on.

The proposed method for virtual prototyping using an avatar has proven to be efficient in the kinematic analysis of rock climbing. Simulating bouldering is quite difficult because it requires high degrees of freedom considering the climber's body and, consequently, a wide range of climbing movements.

We presented different methods for creating a personalised virtual kinematic human model prototype regarding compatibility, the right mix of existing software packages and digital transformation. 
In the first case, we find body areas that are compressed or restructured by the simulated movement or virtual garment. The fact that the avatars zones overlap at a certain point cannot be reproduced accurately. The second case, with 3D to 2D pattern flattening method for sports garments, gives the impression of a viable solution in understanding the anthropometric dimensions through dynamic body movement and extreme postures.

Virtual prototyping based on human kinematic models can be the new go-to method in functional clothing design. Our future work will compare traditionally patterns and patterns obtained with the flattening (model unwrapping) procedure.

Product development designers can communicate faster with textile engineers and deliver more inspiring collections at a lower cost with digital visualisation and prototyping. With 3D technologies in the textile and clothing design industry, the line between "realities" and "virtual" fade even more. A 3D modelling environment provides the logistical advantages of a virtual medium, giving designers free rein when it comes to pursuing perfection. Having the luxury of testing countless iterations of a product without committing (and wasting) endless amounts of resources is also an advantage when it comes to reducing the carbon footprint of the fashion industry.

\section{REFERENCES}

[1] Filipescu, E., Avadanei M., Structura si proiectarea confectiilor textile, Ed. Performantica, Iasi, 2007

[2] Loercher, C., Morlock, S., Schenk A., Motion-oriented 3D analysis of body measurements, In: IOP Conf. Ser.: Mater. Sci. Eng., 2017, 254 172016, https://doi.org/10.1088/1757899X/254/17/172016

[3] Teyeme, Y., Malengier, B., Tesfaye, T., Ciesielska, I., Musa, A., Van Langenhove, L., A Review of Contemporary Techniques for Measuring Ergonomic Wear Comfort of Protective and Sport Clothing, In: Autex Research Journal, 2020, 21, https://doi.org/10.2478/aut-2019-0076

[4] Loercher, C., Morlock, S., Schenk, A., Design of a Motion-Oriented Size System for Optimizing Professional Clothing and Personal Protective Equipment, In: Journal of Fashion Technology \& Textile Engineering, 2018, https://doi.org/10.4172/2329-9568.S4-014

[5] Robinette, K., Blackwell, S., Daanen, H., Boehmer, M., Fleming, S., Brill, T., Hoeferlin, D., Burnsides, D., Anthropometry resource (caesar) final report, volume I: Summary, 2002

[6] Pan, J., Chen, L., Yang, J., Qin, H., Automatic skinning and weight retargeting of articulated characters using extended position-based dynamics, In: The Visual Computer, 2018, 34. 1-13. https://doi.org/10.1007/s00371-017-1413-6

[7] Aburumman, N., Fratarcangeli, M., Skin Deformation Methods for Interactive Character Animation, In: Communications in Computer and Information Science, 2017, 8, 153-174, https://doi.org/10.1007/978-3-319-64870-5_8

[8] SMPL. A Skinned Multi-Person Linear Model, Available at: https://smpl.is.tue.mpg.de/ [Accessed on July 28, 2021]

[9] Dyna. A Model of Dynamic Human Shape in Motion, Available at: http://dyna.is.tue.mpg.de/ [Accessed on July 28, 2021]

[10] Wang, H., Guler, R.A., Kokkinos, I., Papandreou, G., Zafeiriou, S., BLSM: A Bone-Level Skinned Model of the Human Mesh, In: Proceedings of the European Conference on Computer Vision (ECCV), 2020, August

[11] Marcard, T., Henschel, R., Black, M., Rosenhahn, B., Pons-Moll, G., Recovering Accurate 3D Human Pose in the Wild Using IMUs and a Moving Camera, At: $15^{\text {th }}$ European Conference, Munich, Germany, 2018, Proceedings, Part X. https://doi.org/10.1007/978-3-030-01249-6_37

[12] Klepser, A., Pirch, C., Zangue, F., Loercher, C., \& Chen, A., Morlock, S., Is this real? Avatar Generation for 3D Garment Simulation, In: Journal of Textile and Apparel, Technology and Management, 2021

[13] Jolly, K., Krzywinski, S., Rao, P.V.M., Gupta, D., Kinematic modeling of a motorcycle rider for design of functional clothing, In: International Journal of Clothing Science and Technology, 2019, https://doi.org/10.1108/IJCST-02-2019-0020 
[14] Zhang, D., Krzywinski, S., Development of a Kinematic Human Model for Clothing and High Performance Garments, 2019, https://doi.org/68-73. 10.15221/19.068

[15] Legreneur, P., Rogowski, I., Durif, T., Kinematic analysis of the speed climbing event at the 2018 Youth Olympic Games, In: Computer Methods in Biomechanics and Biomedical Engineering, 2019, 22. S264-S266. https://doi.org/10.1080/10255842.2020.1714907

[16] Quaine, F., Martin, L., A biomechanical study of equilibrium in sport rock climbing. Gait and Posture, 1999, 10, 233-239

[17] Hörst, E.J., Training for Climbing: The Definitive Guide to Improving your Climbing Performance, In: Guildford, CT: Falcon Guides, Globe Pequot Press, 2003

[18] 3D Models, Available at: https://www.turbosquid.com/3d-models/ [Accessed on July 28, 2021]

[19] Mixamo, Available at: https://www.mixamo.com/\#/ [Accessed on July 30, 2021]

[20] CLO-3D Fashion Design Software, CLO Official Site, Available at: https://www.clo3d.com [Accessed on July 30, 2021] 